\title{
桥联双萗及其衍生物的合成以及对有机阳离子的络合作用
}

\author{
姚 欢 ${ }^{a, b}$ 孙娇囡 ${ }^{b}$ 柯 华 ${ }^{b}$ 杨留攀 ${ }^{b}$ 李加荣*,a 蒋 伟*,b \\ ( ${ }^{a}$ 北京理工大学化学与化工学院 北京 100081) \\ ( ${ }^{b}$ 南方科技大学化学系 深圳 518055)
}

\begin{abstract}
摘要 对基于 2 -菜酚和丙二缩醛的桥联双菜进行了改造, 合成了六种具有不同取代基、侧壁或桥的桥联双荥衍生物. 通 过单晶衍射和分子模拟对其结构进行了研究, 所有化合物都具有弧形结构. 静电势能表面表明这些弧形分子的内部是 富电子的, 因此能够通过阳离子- $\pi$ 相互作用与有机阳离子发生络合. 通过 ${ }^{1} \mathrm{H} N \mathrm{NR}$ 滴定和 Job's 曲线研究了这些弧形分 子与基于 1,4-二氮杂二环[2.2.2]辛烷(DABCO)的有机阳离子的键合计量比和络合常数. 结果表明富电子的分子对有机 阳离子的络合能力更强，而减少桥上碳原子的数量会减弱分子的络合能力. 这些新型的弧形分子为构建大环受体提供 了很好的骨架单元.
\end{abstract}

关键词 主客体化学; 大环化学; 桥联双荎

\section{Synthesis of Bis-naphthalene and Their Derivatives and Their Complexation with Organic Cation}

\author{
Yao, Huan ${ }^{a, b}$ \\ Sun, Jiaonan ${ }^{b}$ \\ $\mathrm{Ke}, \mathrm{Hua}^{b}$ \\ Li, Jiarong ${ }^{*, a}$ \\ Jiang, Wei*,b \\ Yang, Liupan ${ }^{b}$ \\ $\left({ }^{a}\right.$ School of Chemistry and Chemical Engineering, Beijing Institute of Technology, Beijing 100081) \\ ( ${ }^{b}$ Department of Chemistry, South University of Science and Technology of China, Shenzhen 518055)
}

\begin{abstract}
In this research, the structure of the bis-naphthalene molecular resulting from 2-naphthol and 1,1,1',1'- tetramethoxypropane was modified, and six derivatives with different substituents, sidewalls or bridges were synthesized. Their structures were studied by X-ray crystallography and molecular modelling, and all possess curved architectures. Electrostatic potential energy surfaces show that their inner cavities are electron-rich, and may complex with organic cations through cation- $\pi$ interactions. Their binding stoichiometry and association constants with the 1,4-diazabicyclo[2.2.2]octane (DABCO)-based organic cation were studied by ${ }^{1} \mathrm{H}$ NMR titration and Job's plot. The results show that electron-rich molecules have much stronger association, and reducing the carbon atom in the bridge significantly decreases their association ability. These novel curved structures may work as building blocks for the construction of new macrocyclic receptors.
\end{abstract}

Keywords host-guest chemistry; macrocyclic chemistry; bis-naphthalene

主客体化学是有机超分子化学研究的主要内容之 - $^{[1]}$, 探索新型的主体分子对推动这一领域的发展具有 重要的意义. 具有一定弧度的刚性分子 ${ }^{[2]}$ 是很好的大环 受体基元，其本身就能够识别特定的客体分子 ${ }^{[3]}$. 如 Kagan's 醚 ${ }^{[4]}$ 、Tröger's 碱 ${ }^{[5]}$ 和三蝶烯 ${ }^{[6]}$ 等, 因其固定的构 型和裂穴，被广泛用于构建人工分子钳受体. 同时，还 可以作为模块基元构建大环受体. 如陈传峰等 ${ }^{[6]}$ 利用三 蝶烯分子多样的取代位点, 合成了三(冠醚)、杯芳烃、
内酰胺大环等大环受体以及结构更加复杂的锁烃、分子 笼等. 因此，探索发现新的弧形分子势必能够为超分子 主体的设计合成提供新基元，为有机超分子化学的发展 注入新的内容.

我们的研究兴趣主要集中在菜基超分子受体的设 计、合成及应用 ${ }^{[7 \sim 12]}$. 发现基于刚性桥联双芸的化合物 1 是很好的大环构筑基元. 该化合物最初是由 Whitesides 等 ${ }^{[13]}$ 以 2-薺酚为原料, 在三氟乙酸催化作用

\footnotetext{
* Corresponding authors. E-mail: jiangw@sustc.edu.cn; jrli@bit.edu.cn

Received December 8, 2016; revised December 27, 2016; published online December 30, 2016.

Project supported by the National Natural Science Foundation of China (Nos. 21302090, 21572097) and the Thousand Talents Program-Youth.

国家自然科学基金(Nos. 21302090, 21572097)和中组部 “青年千人计划”资助项目.
} 
下与丙二缩醛反应而成. 这一化合物的空间结构存在一 定的弧形弯曲，因此 Glass 等 ${ }^{[14]}$ 使用该分子构建了苂光 分子管. 我们对 1 的合成方法进行了改进 ${ }^{[9]}$. 在反应溶 剂中加入了四氢呋喃(THF), 使底物的溶解性增加, 扩 展了底物的适用范围, 得到了化合物 $\mathbf{1}$ 的衍生物以及手 性分子钳(Eq. 1, 图 1). 我们还使用该弧形分子构建了 一系列大环受体, 包括动态亚胺大环分子 ${ }^{[10]}$ 和内修饰 分子管 ${ }^{[11]}$, 利用后者实现了在非极性溶剂中对中性分子 的选择性识别. 最近, 我们 ${ }^{[12]}$ 还报道了基于该弧形分子 的水溶性内修饰分子管, 在水中利用氢键实现了对中性 亲水溶剂分子的选择性识别. 该类分子管具有苂光, 而 且能够对地下水中的持久性污染物和致癌物—— 1,4 二 氧六环进行荧光检测, 检测限可达 $119 \mu \mathrm{g} \cdot \mathrm{L}^{-1}$, 接近于 世界卫生组织对饮用水中 $1,4-$ 二氧六环安全线的规定 $\left(50 \mu \mathrm{g} \cdot \mathrm{L}^{-1}\right)$.
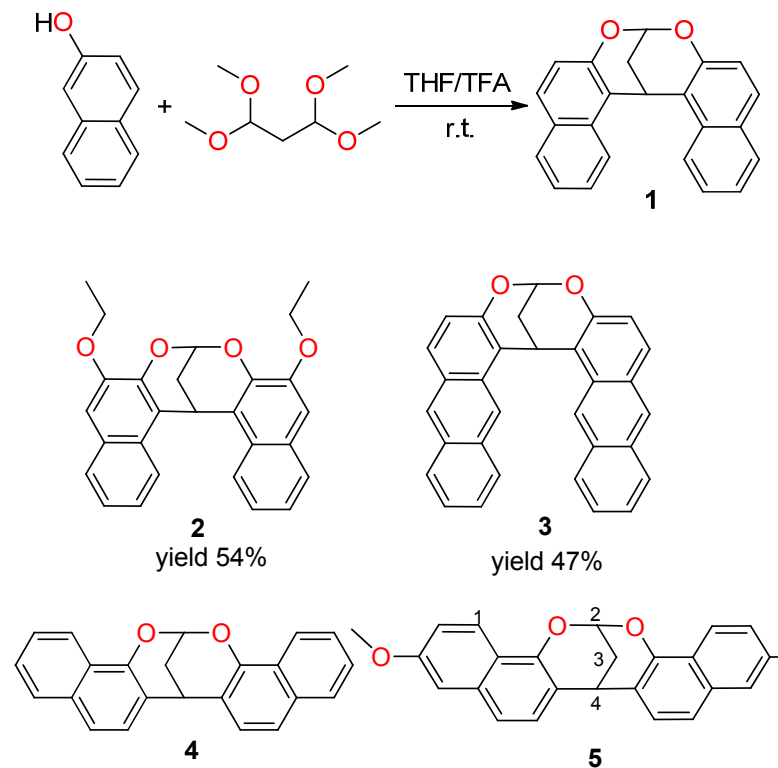

yield $26 \%$
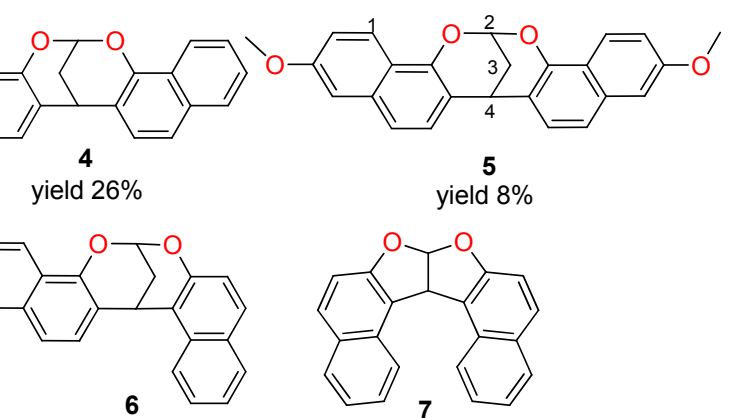

yield $10 \%$
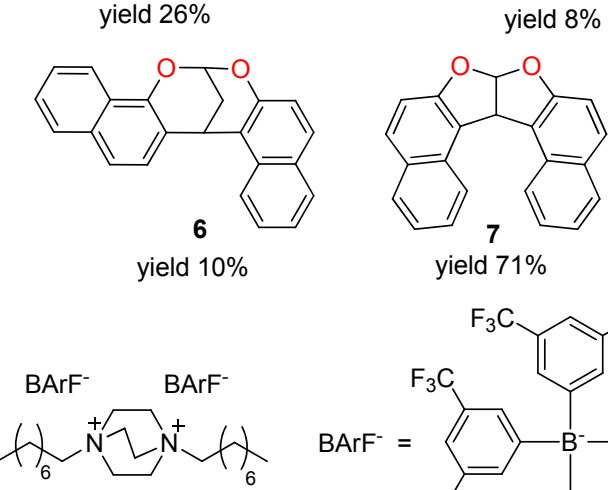

8D8·2BArF

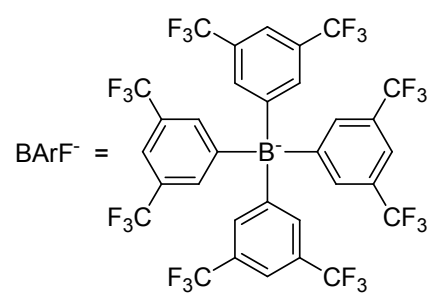

图 1 化合物 $2 \sim 7$ 和阳离子客体 $8 \mathrm{D} 8 \cdot 2 \mathrm{BArF}$ 的结构

Figure 1 Structures of compounds $2 \sim 7$ and cation guest $8 \mathrm{D} 8 \cdot 2 \mathrm{BArF}$
具有弧形结构的桥联双萗 1 是构建大环受体的优良 基元. 若是对这一化合物的结构进行改造，那么所得到 的新型弧形分子可以用于更加多样的大环受体. 本工作 将从三个方面对化合物 $\mathbf{1}$ 的结构进行改造: (1)在化合物 1 的荎环增加取代基或扩展其芳香环; (2)使用不同的荎 酚作为反应原料, 例如用 1-慕酚代替 2-慕酚; (3)对在化 合物 1 中间的桥进行改造，改变桥上碳原子的数量. 同 时, 我们还将研究新获得的弧形分子的主客体化学.

\section{1 结果与讨论}

我们使用 1 的合成条件, 合成了 $2 \sim 7$ 这六种分子 (图 1). 为了在化合物 $\mathbf{1}$ 的基础上改变其侧壁, 分别使用 3-甲氧基-2-蒜酚、2-葱酚、1-菜酚和 6-甲氧基-1-菜酚代 替 2-䒺酚，以 $2: 1$ 的比例与丙二缩醛进行反应，得到了 化合物 $\mathbf{2} \sim \mathbf{5}$; 将 1-荎酚、2-萗酚和丙二缩醛以 $1: 1: 1$ 的比例反应，合成了两边侧壁不对称的分子 $\mathbf{6}$; 为了改 变化合物 1 中间的桥, 用乙二醛代替丙二缩醛与 2-荎酚 反应，得到了桥上为 2 个碳的化合物 7. 这六种化合物 中, 有 1-菜酚参与反应的产率相对来说比较低, 尤其是 化合物 5 的产率只有 $8 \%$. 可能是因为 1-䒺酚导致更多 的副反应

将化合物 $\mathbf{5}$ 的二氯甲烷(DCM)/MeOH 溶液在室温 下缓慢挥发, 我们得到了化合物 5 的晶体结构(图 2a). 在晶体中, 化合物 $\mathbf{5}$ 形成了相对刚性的弧形结构. 通过 能量最优化计算，我们得到了这七种分子的模型，发现 它们都具有一定的弧度(图 2b). 它们的静电势能表面显 示弧形分子内部都是富电子的(图 2c). 由此推测, 它们 或许能够通过阳离子 $-\pi$ 相互作用，与有机阳离子客体发 生主客体络合.

正如之前报道的 ${ }^{[9]}$, 我们选择了有机阳离子客体 $8 \mathrm{D} 8 \cdot 2 \mathrm{BArF}$ (图 1)来进行主客体识别. 如图 3 所示, 当将 主体 5 与客体 $8 \mathrm{D}^{2+}$ 以 $1: 1$ 的比例在 $\mathrm{CD}_{2} \mathrm{Cl}_{2}$ 中混合, 它 们的质子峰都发生了一定的位移. 尤其是客体上 $\mathrm{DABCO}$ 的质子 $\mathrm{a}$ 向高场位移了 0.294 , 而烷基链上的质 子位移相对较小. 这说明客体的 $\mathrm{DABCO}$ 部分与主体发 生了络合, 而不是烷基链. 当 $\mathrm{DABCO}$ 络合在弧形空腔 内, 经历了两个䒬环的屏蔽效应, 所以向高场位移. 其 它的弧形分子与 $8 \mathrm{D} 8^{2+}$ 的键合也有类似的现象.

接下来，从化合物 5 与 $8 \mathrm{D} 8^{2+}$ 的 Job's 曲线可知它们 之间络合的化学计量比为 $1: 1$ (图 4a). 因此, 利用核磁 滴定实验，通过监测化合物 5 上的质子 3 的化学位移, 并对其进行非线性拟合，可以测得其络合常数为 107.6 $\mathrm{L} \cdot \mathrm{mol}^{-1}$ (图 4b). 利用相同的方法, 也可以测得其它几种 弧形分子与 $8 \mathrm{D} 8^{2+}$ 的络合常数, 结果列于表 1 中. 
a)

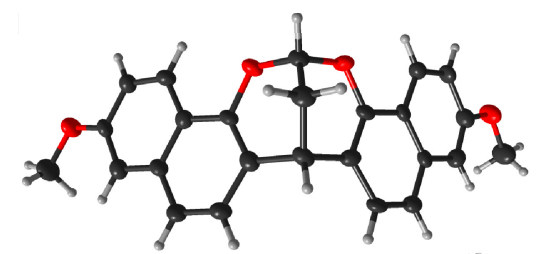

b)

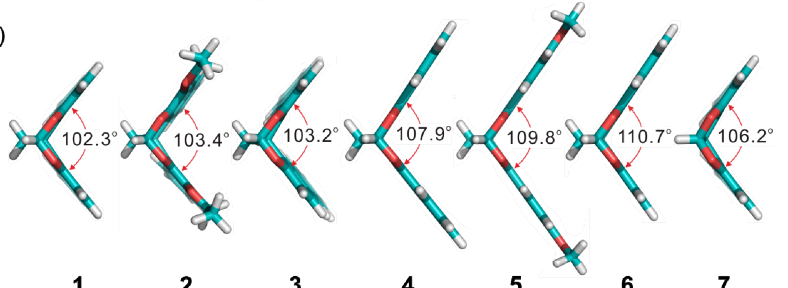

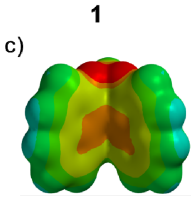
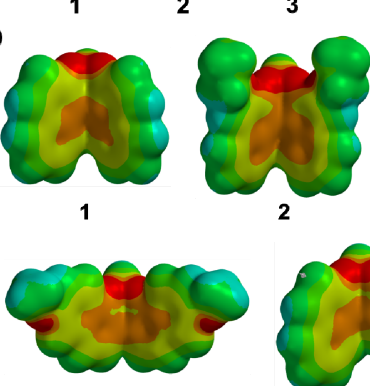

5
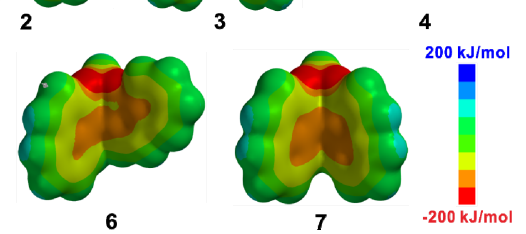

图 2 (a) 化合物 $\mathbf{5}$ 的单晶结构、(b)化合物 $\mathbf{1} \sim 7$ 能量最优化结 构的二面角及(c)化合物 1 7 的理论计算静电势能表面

Figure 2 (a) The single crystal structure of compound 5, (b) dihedral angles of energy-minimized structures of compounds $1 \sim 7$ and (c) electrostatic potential energy surfaces of compounds $1 \sim 7$ All the computations were performed at the AM1 level of theory by using Spartan'14 (Wavefunction, Inc.)

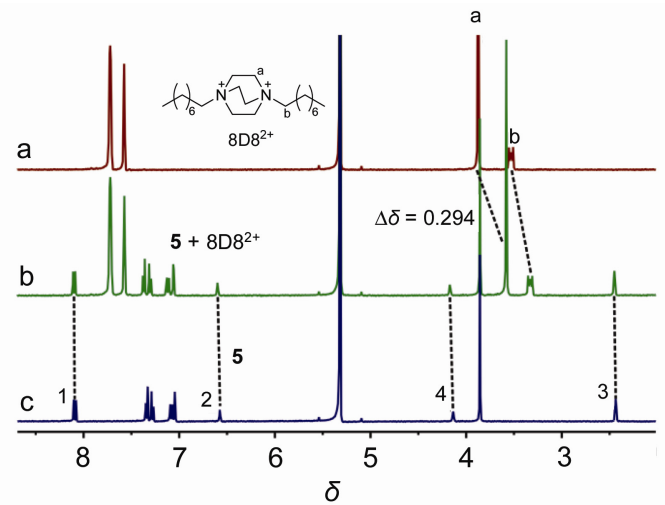

图 3 (a)客体 $8 \mathrm{D} 8^{2+}\left(2.0 \mathrm{mmol} \cdot \mathrm{L}^{-1}, \mathrm{CD}_{2} \mathrm{Cl}_{2}\right)$ 、(b)主客体 $1: 1$ 混合物 $\left(2.0 \mathrm{mmol} \cdot \mathrm{L}^{-1}, \mathrm{CD}_{2} \mathrm{Cl}_{2}\right)$ 及(c)主体 $5\left(2.0 \mathrm{mmol} \cdot \mathrm{L}^{-1}\right.$, $\mathrm{CD}_{2} \mathrm{Cl}_{2}$ ) 的 ${ }^{1} \mathrm{H} \mathrm{NMR}$ 谱

Figure $3{ }^{1} \mathrm{H}$ NMR spectra of (a) $8 \mathrm{D} 8^{2+}$, (b) $1: 1$ mixture and (c) 5

从表中可以看出, 化合物 $\mathbf{2}$ 和 $\mathbf{3}$ 的络合常数都高于 $80 \mathrm{~L} \cdot \mathrm{mol}^{-1}$, 比 1 的 $50.0 \mathrm{~L} \cdot \mathrm{mol}^{-1}$ 大; 5 的络合常数高达

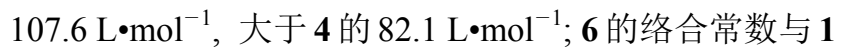
相近，而 7 最小. 这些结果能够通过结合图 $2 \mathrm{a}$ 的静电势 能表面得到解释: 化合物 $\mathbf{2}$ 比 1 增加了给电子的乙氧基
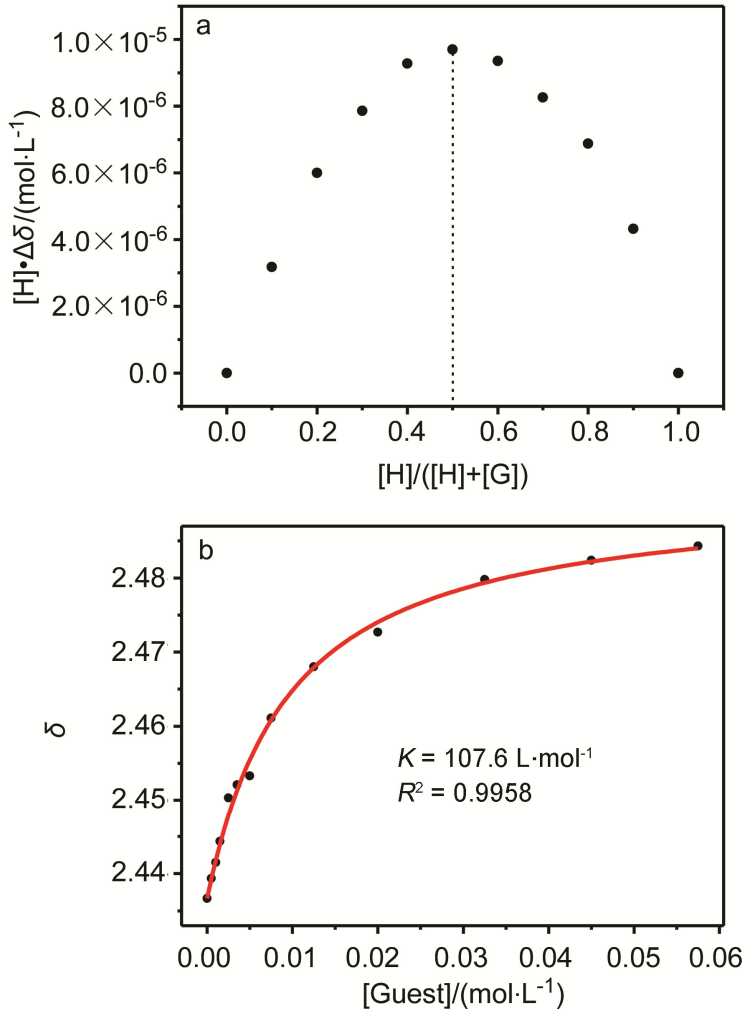

图 4 (a) ${ }^{1} \mathrm{H} \mathrm{NMR}$ 实验测得的 $\mathbf{5}$ 和 $8 \mathrm{D} 8^{2+}$ 络合的 Job's 曲线 $\left([\mathbf{5}]+\left[8 \mathrm{D} 8^{2+}\right]=2.0 \mathrm{mmol} \cdot \mathrm{L}^{-1}\right)$ 及 $(\mathrm{b}) 8 \mathrm{D} 8^{2+}$ 与 $\mathbf{5}$ 核磁滴定氢谱质 子 3 位移的非线性拟合曲线

Figure 4 (a) Job's plot for the complex between 5 and $8 \mathrm{D} 8^{2+}$ as determined by ${ }^{1} \mathrm{H}$ NMR experiments $\left([5]+\left[8 \mathrm{D} 8^{2+}\right]=2.0\right.$ $\mathrm{mmol} \cdot \mathrm{L}^{-1}$ ) and (b) non-linear fitting of the NMR titration curve of 5 by $8 \mathrm{D} 8^{2+}$

The chemical shift of proton 3on $\mathbf{5}$ is monitored

表 1 通过 ${ }^{1} \mathrm{H} \mathrm{NMR}\left(400 \mathrm{MHz}, \mathrm{CD}_{2} \mathrm{Cl}_{2}, 25{ }^{\circ} \mathrm{C}\right)$ 滴定测得的络 合常数(主体分子的浓度都为 $0.5 \mathrm{mmol} \cdot \mathrm{L}^{-1}$ )

Table 1 Binding constants as determined by ${ }^{1} \mathrm{H}$ NMR titration $\left(400 \mathrm{MHz}, \mathrm{CD}_{2} \mathrm{Cl}_{2}, 25{ }^{\circ} \mathrm{C}\right.$ ) (the concentrations of the hosts are fixed at $0.5 \mathrm{mmol} \cdot \mathrm{L}^{-1}$ )

\begin{tabular}{cc}
\hline Compd. & $K_{\mathrm{a}} /\left(\mathrm{L} \cdot \mathrm{mol}^{-1}\right)$ \\
\hline $\mathbf{1}$ & $50.0 \pm 3.0$ \\
$\mathbf{2}$ & $85.0 \pm 5.1$ \\
$\mathbf{3}$ & $82.0 \pm 4.9$ \\
$\mathbf{4}$ & $82.1 \pm 5.3$ \\
$\mathbf{5}$ & $107.6 \pm 7.5$ \\
$\mathbf{6}$ & $58.2 \pm 3.6$ \\
$\mathbf{7}$ & $6.6 \pm 0.1$ \\
\hline
\end{tabular}

取代基，使它更加的富电子，与阳离子客体的相互作用 增强; $\mathbf{3}$ 的侧壁在 $\mathbf{1}$ 的基础上多了一个苯环，使其富电子 表面增大，从而与阳离子客体相互作用的弧形面积增 加; $\mathbf{4}$ 的结构与 1 完全不同, 但它的静电势能表面的富电 子区域面积更大，导致与阳离子客体的作用增强; 同样, 化合物 5 比 4 多了给电子的甲氧基, 增强了空腔内的富 
电性. 它与 $8 \mathrm{D} 8^{2+}$ 的主客体复合物的模拟结构能够看到 明显的 $\mathrm{CH}-\pi$ 相互作用(图 5); 6 的结构是 1 和 4 的结合, 所以络合常数介于两者之间; 化合物 7 中间的桥太小, 导致两个菜环平面二面角变大, 而富电性没有增加, 因 此与阳离子的络合能力最小.
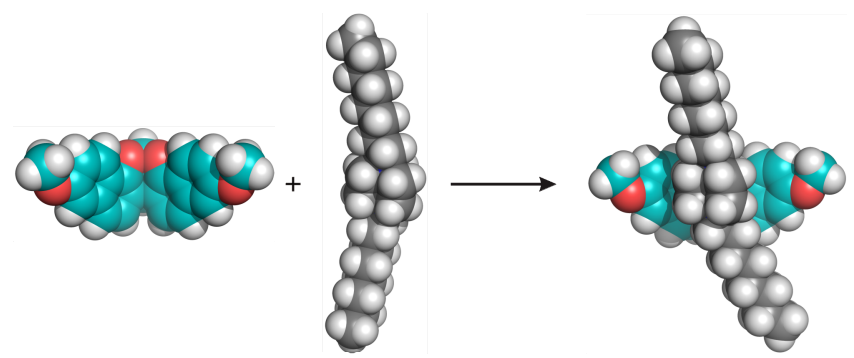

图 5 化合物 5 与 $8 \mathrm{D} 8^{2+}$ 的复合物的能量最优化结构

Figure 5 Energy-minimized structure of the complex between compound $\mathbf{5}$ and $8 \mathrm{D} 8^{2+}$

All the computations were performed at the AM1 level of theory by using Spartan'14 (Wavefunction, Inc.)

\section{2 结论}

我们通过简单的方法合成了 6 种具有一定弧度的刚 性分子. 并且通过 ${ }^{1} \mathrm{H}$ NMR 滴定的方法测定了它们与有 机阳离子客体的络合能力. 这些新型的弧形分子大部分 比已有的大环基元 1 具有更好的络合能力. 而且其弧形 的骨架结构是很好的大环受体的构筑基元. 在未来的研 究中, 我们将利用这些新型的弧形分子构建萗基大环受 体, 进一步丰富大环受体的工具箱.

\section{3 实验部分}

\section{1 仪器与试剂}

薄层色谱(TLC)在 $0.25 \mathrm{~mm}$ 烟台硅胶板(60F-254)上 进行. 柱色谱在硅胶 60(青岛 40～63 nm, 230 400 目) 上进行. ${ }^{1} \mathrm{H}$ NMR 和 ${ }^{13} \mathrm{C}$ NMR 采用 Bruker Avance 400 $\mathrm{MHz}$ 核磁共振波谱仪测定. 高分辨质谱用 Applied Biosystems Elite ESI-QqTOF 质谱仪测定. 2-葱酚是根据文 献[15]方法合成. $7 a, 14 c$-二氢菜并 $[2,1-b]$ 菜并 $\left[1^{\prime}, 2^{\prime}: 4,5\right]$ 呋 喃并 [3,2- $d]$ 呋喃(7)是已知化合物 ${ }^{[16]}$, 这里以本文的方法 重新合成. 其它试剂均为分析纯商品化试剂.

\section{2 实验方法}

3.2.1 6,10-二乙氧基- $16 H-8,16$ - 甲基二蔡并 $[2,1$ $\left.d: 1^{\prime}, 2^{\prime}-g\right][1,3]$ 二氧环辛烷(2)的合成

向装有 3-乙氧基-2-菜酚( $2.00 \mathrm{~g}, 10.6 \mathrm{mmol})$ 的圆底 烧瓶中加入 $18.0 \mathrm{~mL}$ 三氟乙酸/四氢呋喃 $(V: V=2: 1)$ 的混合溶剂. 在搅拌下用注射器将丙二缩醛 $(0.87 \mathrm{~mL}$, $5.3 \mathrm{mmol}$ )逐滴加入上述溶液中, 时间约为 $10 \mathrm{~min}$. 所得 的混合物在常温下继续摚拌 $3 \mathrm{~h}$ 后, 向其加入饱和的
$\mathrm{NaHCO}_{3}$ 水溶液，调 $\mathrm{pH}=7$, 淬灭反应. 之后用二氯甲烷 $(10 \mathrm{~mL} \times 3)$ 萃取. 分离有机相并用无水硫酸钠干燥, 旋 蒸除去溶剂, 得到粗产物. 最后用柱层析 $\left[\mathrm{SiO}_{2}, V(\right.$ 石油 醚)： $V($ 二氯甲烷 $)=6 ： 1$ ] 分离得到 $1.18 \mathrm{~g}$ 棕色固体 2, 产率 54\%. m.p. 250 251 ${ }^{\circ} \mathrm{C}$; ${ }^{1} \mathrm{H}$ NMR $(400 \mathrm{MHz}$, $\left.\mathrm{CDCl}_{3}\right) \delta: 8.55(\mathrm{~d}, J=8.5 \mathrm{~Hz}, 2 \mathrm{H}), 7.63(\mathrm{dd}, J=8.1,1.2$ $\mathrm{Hz}, 2 \mathrm{H}), 7.45$ (ddd, $J=8.3,6.9,1.3 \mathrm{~Hz}, 2 \mathrm{H}), 7.32$ (dd, $J=$ $8.1,6.9 \mathrm{~Hz}, 2 \mathrm{H}), 7.02(\mathrm{~s}, 2 \mathrm{H}), 6.58$ (t, $J=1.8 \mathrm{~Hz}, 1 \mathrm{H}), 5.41$ $(\mathrm{q}, J=2.7 \mathrm{~Hz}, 1 \mathrm{H}), 4.33 \sim 4.09(\mathrm{~m}, 4 \mathrm{H}), 2.46(\mathrm{t}, J=2.6$ $\mathrm{Hz}, 2 \mathrm{H}), 1.56(\mathrm{t}, J=7.0 \mathrm{~Hz}, 6 \mathrm{H}) ;{ }^{13} \mathrm{C} \mathrm{NMR}(101 \mathrm{MHz}$, $\left.\mathrm{CDCl}_{3}\right) \delta: 148.03,142.67,130.04,127.48,126.27,124.14$, 124.00, 122.78, 119.81, 107.05, 91.54, 64.28, 26.54, 22.96, 14.81; ESI-TOF-HRMS calcd for $\mathrm{C}_{27} \mathrm{H}_{25} \mathrm{O}_{4}[\mathrm{M}+\mathrm{H}]^{+}$ 413.1743, found 413.1744 .

$3.2 .219 H-9,19$-甲基二葱并 $\left[2,1-d: 1^{\prime}, 2^{\prime}-g\right][1,3]$ 二氧环 辛烷(3)的合成

使用与化合物 2 相同的合成方法，以 2-葱酚和丙二 缩醛为原料得到白色固体 3, 产率 36\%. m.p. 298 $299{ }^{\circ} \mathrm{C} ;{ }^{1} \mathrm{H}$ NMR (400 MHz, $\mathrm{CDCl}_{3}$ ) $\delta: 9.38$ (s, 2H), 8.31 (s, 2H), 8.14 (d, $J=8.5 \mathrm{~Hz}, 2 \mathrm{H}), 7.93$ (d, $J=8.4 \mathrm{~Hz}, 2 \mathrm{H})$, $7.80(\mathrm{~d}, J=9.1 \mathrm{~Hz}, 2 \mathrm{H}), 7.52$ (ddd, $J=8.3,6.6,1.2 \mathrm{~Hz}$, 2H), 7.42 (ddd, $J=8.0,6.6,1.2 \mathrm{~Hz}, 2 \mathrm{H}$ ), 7.23 (d, $J=9.1$ $\mathrm{Hz}, 2 \mathrm{H}), 6.42$ (q, $J=2.0 \mathrm{~Hz}, 1 \mathrm{H}), 5.71$ (s, 1H), 2.62 (dd, $J=3.2,2.1 \mathrm{~Hz}, 2 \mathrm{H}) ;{ }^{13} \mathrm{C} \mathrm{NMR}\left(101 \mathrm{MHz}, \mathrm{CDCl}_{3}\right) \delta$ : $149.91,131.94,130.10,129.98,129.13,128.97,128.10$, $127.91,127.34,126.14,124.96,121.35,119.74,117.16$, 91.88, 29.87, 27.07; ESI-TOF-HRMS calcd for $\mathrm{C}_{31} \mathrm{H}_{21} \mathrm{O}_{2}$ $[\mathrm{M}+\mathrm{H}]^{+}$425.1536, found 425.1530.

$3.2 .37 H-7,15$-甲基二荟并 $\left[1,2-d: 2^{\prime}, 1^{\prime}-g\right][1,3]$ 二氧环 辛烷(4)的合成

使用与化合物 2 相同的合成方法，以 1-菱酚和丙二 缩醛为原料得到白色固体 4, 产率 26\%. m.p. 204 $205{ }^{\circ} \mathrm{C} ;{ }^{1} \mathrm{H}$ NMR (400 MHz, $\left.\mathrm{CDCl}_{3}\right) \delta$ : 8.27 8.21 (m, $2 \mathrm{H}), 7.74 \sim 7.68(\mathrm{~m}, 2 \mathrm{H}), 7.46 \sim 7.42(\mathrm{~m}, 2 \mathrm{H}), 7.42 \sim 7.39$ (m, 2H), 7.37 (d, $J=2.0 \mathrm{~Hz}, 4 \mathrm{H}), 6.63$ (p, $J=2.1 \mathrm{~Hz}, 1 \mathrm{H})$, $4.18(\mathrm{td}, J=2.9,1.6 \mathrm{~Hz}, 1 \mathrm{H}), 2.47(\mathrm{dd}, J=3.0,2.1 \mathrm{~Hz}$, $2 \mathrm{H}) ;{ }^{13} \mathrm{C} \mathrm{NMR}\left(101 \mathrm{MHz}, \mathrm{CDCl}_{3}\right) \delta: 146.48,133.64$, 127.62, 126.06, 125.68, 125.49, 124.66, 121.85, 121.08, 121.05, 92.69, 32.00, 26.00; ESI-TOF-HRMS calcd for $\mathrm{C}_{23} \mathrm{H}_{17} \mathrm{O}_{2}[\mathrm{M}+\mathrm{H}]^{+}$325.1229, found 325.1222.

$3.2 .43,11$ - 二甲氧基 - $7 H-7,15$ - 甲基二荅并 [1,2$\left.d: 2^{\prime}, 1^{\prime}-g\right][1,3]$ 二氧环辛烷(5)的合成

使用与化合物 2 相同的合成方法，以 6-甲氧基-1菜酚和丙二缩醛为原料得到白色固体 5 , 产率 $8 \%$. m.p. $251 \sim 252{ }^{\circ} \mathrm{C} ;{ }^{1} \mathrm{H}$ NMR (400 MHz, $\left.\mathrm{CDCl}_{3}\right) \delta: 8.14(\mathrm{~d}, J=$ 
$9.2 \mathrm{~Hz}, 2 \mathrm{H}), 7.31$ (d, $J=8.4 \mathrm{~Hz}, 2 \mathrm{H}), 7.27$ (s, 4H), 7.09 (dd, $J=9.2,2.5 \mathrm{~Hz}, 2 \mathrm{H}), 7.01(\mathrm{~d}, J=2.5 \mathrm{~Hz}, 2 \mathrm{H}), 6.58$ (q, $J=2.0 \mathrm{~Hz}, 1 \mathrm{H}), 4.11$ (q, $J=2.8 \mathrm{~Hz}, 1 \mathrm{H}), 3.87(\mathrm{~s}, 6 \mathrm{H})$, $2.45 \sim 2.41(\mathrm{~m}, 2 \mathrm{H}) ;{ }^{13} \mathrm{C}$ NMR $\left(101 \mathrm{MHz}, \mathrm{CDCl}_{3}\right) \delta$ : $157.90,146.61,135.00,126.17,123.53,119.90,119.41$, $118.12,105.92,92.75,55.38,31.65,29.86,26.26$; ESITOF-HRMS calcd for $\mathrm{C}_{25} \mathrm{H}_{21} \mathrm{O}_{4}[\mathrm{M}+\mathrm{H}]^{+} 385.1434$, found 385.1430 .

$3.2 .514 H-6,14$-甲基二䓠并 $\left[1,2-d: 1^{\prime}, 2^{\prime}-g\right][1,3]$ 二氧环 辛烷(6)的合成

使用与化合物 2 相同的合成方法, 以 1-菜酚、2-萫 酚和丙二缩醛为原料得到白色固体 6, 产率 $10 \%$. m.p. 196 $197{ }^{\circ} \mathrm{C} ;{ }^{1} \mathrm{H}$ NMR $\left(400 \mathrm{MHz}, \mathrm{CDCl}_{3}\right) \delta: 8.41(\mathrm{~d}, J=$ $8.5 \mathrm{~Hz}, 1 \mathrm{H}), 8.25$ (ddd, $J=8.3,1.6,0.8 \mathrm{~Hz}, 1 \mathrm{H}), 7.76(\mathrm{dt}$, $J=8.1,0.8 \mathrm{~Hz}, 1 \mathrm{H}), 7.71 \sim 7.68(\mathrm{~m}, 1 \mathrm{H}), 7.66 \sim 7.55(\mathrm{~m}$, $3 \mathrm{H}), 7.48 \sim 7.32(\mathrm{~m}, 4 \mathrm{H}), 7.16(\mathrm{~d}, J=8.9 \mathrm{~Hz}, 1 \mathrm{H}), 6.49(\mathrm{~d}$, $J=1.8 \mathrm{~Hz}, 1 \mathrm{H}), 4.88(\mathrm{~d}, J=1.8 \mathrm{~Hz}, 1 \mathrm{H}), 2.53 \sim 2.37(\mathrm{~m}$, $2 \mathrm{H}) ;{ }^{13} \mathrm{C}$ NMR $\left(101 \mathrm{MHz}, \mathrm{CDCl}_{3}\right) \delta: 149.09,146.89$, 133.44, 131.01, 129.74, 129.02, 128.25, 127.56, 126.87, $126.15,125.71,125.57,124.66,123.76,121.84,120.81$, $120.39,119.26,118.45,92.13,29.86,26.20,25.91$; ESITOF-HRMS calcd for $\mathrm{C}_{23} \mathrm{H}_{17} \mathrm{O}_{2}[\mathrm{M}+\mathrm{H}]^{+} 325.1223$, found 325.1220 .

3.2.67 7a, 14c-二氢萮并 $[2,1-b]$ 萗并 $\left[1^{\prime}, 2^{\prime}: 4,5\right]$ 呋喃并 $[3,2-d]$ 呋喃(7)的合成

使用与化合物 $\mathbf{2}$ 相同的合成方法, 以 2-荎酚和乙二 酲为原料得到白色固体 $7^{[16]}$, 产率 $71 \%$. m.p. 227 $229{ }^{\circ} \mathrm{C} ;{ }^{1} \mathrm{H}$ NMR $\left(400 \mathrm{MHz}, \mathrm{CD}_{2} \mathrm{Cl}_{2}\right) \delta: 8.31$ (d, $J=8.4$ $\mathrm{Hz}, 2 \mathrm{H}), 7.83$ (dd, $J=8.3,1.1 \mathrm{~Hz}, 2 \mathrm{H}), 7.77$ (d, $J=8.8 \mathrm{~Hz}$, $2 \mathrm{H}$ ), 7.54 (ddd, $J=8.3,6.9,1.3 \mathrm{~Hz}, 2 \mathrm{H}), 7.35(\mathrm{~s}, 2 \mathrm{H}), 7.23$ (d, $J=8.8 \mathrm{~Hz}, 2 \mathrm{H}), 7.13(\mathrm{~d}, J=5.9 \mathrm{~Hz}, 1 \mathrm{H}), 5.64$ (d, $J=$ $6.0 \mathrm{~Hz}, 1 \mathrm{H}) ;{ }^{13} \mathrm{C}$ NMR $\left(101 \mathrm{MHz}, \mathrm{CDCl}_{3}\right) \delta: 156.61$, $130.87,130.61,130.43,129.43,127.09,123.79,123.51$, 118.81, 115.10, 112.42, 49.72; ESI-TOF-HRMS calcd for $\mathrm{C}_{22} \mathrm{H}_{15} \mathrm{O}_{2}[\mathrm{M}+\mathrm{H}]^{+}$311.1067, found 311.1063.

辅助材料 (Supporting Information) 化合物的 ${ }^{1} \mathrm{H}$ $\mathrm{NMR},{ }^{13} \mathrm{C} \mathrm{NMR}$ 谱、高分辨质谱、单晶结构数据、主客 体 $1: 1{ }^{1} \mathrm{H}$ NMR 谱、络合常数 ${ }^{1} \mathrm{H}$ NMR 滴定谱图及络 合曲线. 这些材料可以免费从本刊网站(http://siocjournal.cn/)上下载.

\section{References}

[1] (a) Tian, J.; Chen, L.; Zhang, D. W.; Liu, Y.; Li, Z. T. Chem.
Commun. 2016, 52, 6351.

(b) Chi, X.; Yu, G.; Shao, L.; Chen, J.; Huang, F. J. Am. Chem. Soc. 2016, 138, 3168 .

(c) Gao, B.; Tan, L. L.; Song, N.; Li, K.; Yang, Y. W. Chem. Commun. 2016, 52, 5804.

(d) Zhang, W.; Zhang, Y. M.; Li, S. H.; Cui, Y. L.; Yu, J.; Liu, Y. Angew. Chem., Int. Ed. 2016, 128, 11624.

(e) Ma, J.; Meng, Q.; Hu, X.; Li, B.; Ma, S.; Hu, B.; Li, C. Org. Lett. 2016, 18, 5740.

(f) Chen, H.; Fan, J.; Hu, X.; Ma, J.; Wang, S.; Li, J.; Li, C. Chem. Sci. 2015, 6, 197.

(g) Wang, X.; Han, K.; Li, J.; Jia, X.; Li, C. Polym. Chem. 2013, 4, 3998.

(h) Wang, Y.; Ping, G.; Li, C. Chem. Commun. 2016, 52, 9858.

[2] (a) Zhou, C. E.; Zhao, Z. G.; Tang, X. L. Chin. J. Org. Chem. 2007, 27, 513 (in Chinese).

(周彩娥, 赵志刚, 唐晓丽, 有机化学, 2007, 27, 513.)

(b) Peng, Y.; Mou, Q. M.; Yang, Z. X.; Chen, S. H. Chin. J. Org. Chem. 2004, 24, 399 (in Chinese).

(彭游, 牟其明, 杨祖幸, 陈淑华, 有机化学, 2004, 24, 399.)

[3] (a) Rebek, J. Science 1987, 235, 1478.

(b) Rebek, J. Pure Appl. Chem. 1989, 61, 1517.

[4] Harmata, M. Acc. Chem. Res. 2004, 37, 862.

[5] Dolenský, B.; Havlík, M.; Král, V. Chem. Soc. Rev. 2012, 41, 3839.

[6] (a) Han, T.; Chen, C. F. Org. Lett. 2006, 8, 1069.

(b) Chen, C. F. Chem. Commun. 2011, 47, 1674.

(c) Jiang, Y.; Chen, C. F. Eur. J. Org. Chem. 2011, 32, 6377.

(d) Meng, Z.; Han, Y.; Wang, L. N.; Xiang, J. F.; He, S. G.; Chen, C. F. J. Am. Chem. Soc. 2015, 137, 9739.

[7] Yang, L. P.; Liu, W. E.; Jiang, W. Tetrahedron Lett. 2016, 57, 3978.

[8] (a) Jia, F.; He, Z.; Yang, L. P.; Pan, Z. S.; Yi, M.; Jiang, R. W.; Jiang, W. Chem. Sci. 2015, 6, 6731.

(b) Jia, F.; Wang, H. Y.; Li, D. H.; Yang, L. P.; Jiang, W. Chem. Commun. 2016, 52, 5666.

(c) Jia, F.; Li, D. H.; Yang, T. L.; Yang, L. Pan.; Dang, L.; Jiang, W. Chem. Commun. 2017, 53, 336.

(d) Yang, L. P.; Jia, F.; Zhou, Q. H.; Pan, F.; Sun, J. N.; Rissanen, K.; Chung, L. W.; Jiang, W. Chem.-Eur. J. 2017, 23, 1516.

[9] He, Z.; Yang, X.; Jiang, W. Org. Lett. 2015, 17, 3880.

[10] (a) He, Z.; Ye, G.; Jiang, W. Chem. Eur. J. 2015, 21, 3005.

(b) Huang, G. B.; Jiang, W. Prog. Chem. 2015, 27, 744 (in Chinese).

(黄国宝, 蒋伟, 化学进展, 2015, 27, 744.)

[11] (a) Huang, G.; He, Z.; Cai, C. X.; Pan, F.; Yang, D.; Rissanen, K.; Jiang, W. Chem. Commun. 2015, 51, 15490.

(b) Huang, G.; Valkonen, A.; Rissanen, K.; Jiang, W. Chem. Commun. 2016, 52, 9078.

[12] Huang, G. B.; Wang, S. H.; Ke, H.; Yang, L. P.; Jiang, W. J. Am. Chem. Soc. 2016, 138, 14550.

[13] Van Allan, J. A.; Giannini, D. D.; Whitesides, T. H. J. Am. Chem. Soc. 1982, 47, 820 .

[14] Shorthill, B. J.; Avetta, C. T.; Glass, T. E. J. Am. Chem. Soc. 2004, 126, 12732.

[15] Geiseler, O.; Müller, M.; Podlech, J. Tetrahedron 2013, 69, 3683.

[16] Kito, T.; Yoshinaga, K.; Yamaye, M.; Mizobe, H. J. Org. Chem. 1991, 56, 3336. 